\title{
Research on the Influence of Coating Solid on Binder Migration
}

\author{
Ying $\mathrm{Li}^{1,}$, Jie Zhang $^{1}$, Wen juan $\mathrm{Gu}^{1}$ and Banggui $\mathrm{He}^{1}$ \\ ${ }^{1}$ Faculty of Mechanical and Electrical Engineering, Kunming University of Science and Technology, \\ Kunming, 650093, China \\ aEmail:haishanying@126.com
}

Keywords: Binder migration; Coating solid; Base paper; Coating surface; SEM; LSCM

\begin{abstract}
The information of surface topography and structure of coating layer were significant to the chemical-physical properties of coated paper as well as the print quality The main aim of this study was to investigate the effect of coating solid on the binder distribution in the method of numerical analysis by Laser Scanning Confocal Microscope (LSCM). In this study, Rhodamine B was used to stain the binder and as the probe to characterize z-directional distribution of the binder using LSCM. It could be concluded that the coating solid played an important effect on binder distribution in coated paper and low coating solid with more water accelerated binder migration to the interior of base paper and coating surface. The appropriate coating solid is good to binder distribution and coating layer forming.
\end{abstract}

\section{Introduction}

Binder is the essential component of coating color recipe. The amount and type of latex binder has a significant effect on both the structure and chemical-physical properties of coated paper, which affect critical surface properties, e.g., print gloss, roughness, ink setting rate via liquid absorption, and print mottle[1-2]. The binder was found to be significantly affected coating structure and print quality based on AFM phase imaging and TOF-SIMS mapping [3, 4]. Binder migration towards base paper will contribute to the ink absorption and ink usage increasing ink absorption, and decrease printing gloss. In some researches, some treatments must be done before measurements, which might destroy paper structure and do some disadvantages to measurements [5, 6]. In this study, Rhodamine B was used to stain the binder and as the probe to characterize z-directional distribution of the binder using LSCM without any destroy. The main aim of this investigation was to characterize the z-directional distribution of the binder and obtain the influence of coating solid of coated paper on the binder migration.

\section{Experimental}

Dying.Rhodamine B (Sigma-R6626, Co. Ltd) was used as fluorescent dye for staining with the binder because it was successfully applied in some studies [7, 8]. Rhodamine B was dissolved in ethanol to reach $0.05 w t \%$. $0.05 w t \%$ of Rhodamine B was added to carboxylic styrene-butadiene latex, and then it was stirred. The binder was held to stain completely for $24 \mathrm{~h}$ before coating in order to make Rhodamine B and binder become the whole.

Coating. The basis weight of uncoated base paper (supplied by Dong Tang Paper Mill) was $70 \mathrm{~g} / \mathrm{m}^{2}$.The coatings of paper were composed with kaolin pigment (supplied by Mao Ming Clay Company, China) and carboxylic styrene-butadiene latex (supplied by BASF Company, China). The coating components are indicated in Table 1. Coating was performed with a bar coater (model K303 Multi-coater, RK Print Coat Instruments Ltd, United Kingdom). The coated paper was moved to a drying oven for $1 \mathrm{~min}$ with the drying temperature $110^{\circ} \mathrm{C}$.

- Coating speed $=5 \mathrm{~m} / \mathrm{min}$

- Drying temperature $=110^{\circ} \mathrm{C}$

- Drying time $=1 \mathrm{~min}$

- Coating thickness $=6 \mu \mathrm{m}$ 
Table 1 Coating color recipes

\begin{tabular}{ccccc}
\hline Sample & Kaolin $\left(\mathrm{pph}^{*}\right)$ & $\mathrm{SB}\left(\mathrm{pph}^{*}\right)$ & $\begin{array}{c}\text { Coating } \\
\text { Thickness }(\mu \mathrm{m})\end{array}$ & Coating Solid (\%) \\
\hline H1 & 100 & 15 & 6 & 45 \\
H2 & 100 & 15 & 6 & 50 \\
H3 & 100 & 15 & 6 & 55 \\
H4 & 100 & 15 & 6 & 60 \\
\hline
\end{tabular}

*Parts per hundred

Measurements of Scanning Electron Microscopy. Scanning electron microscopy (Hitachi S3700N, Japan) was utilized to investigate the elemental composition of the coatings surface. For SEM observation, all coating samples were gold coated and $20 \mathrm{KV}$ beam energy was used to get the information of elemental composition of the coatings.

Laser Scanning Confocal Microscopy. A Laser Scanning Confocal Microscopy (Leica TCS-SP5) was used to obtain the z-directional distribution of stained binder. Immersion liquid was supplied by Leica. An X40 oil-immersion objective lens (HC PLAPO, NA 1.25) was chosen. As the laser beam scanned the ink film, the emitted fluorescent light was detected by a photodetector. A confocal beam splitter filter (DD458/514) was used to separate the fluorescent light. The second PMT received He-Ne 514 laser signal, and the detected wavelength ranged from $520 \mathrm{~nm}$ to $615 \mathrm{~nm}$. The appropriate focus plane and the best image could be obtained by adjusting the z-directional position, PMT gain, and PMT offset, respectively. Three-dimensional information was gathered as the optical depth images obtained from the scanner. Confocal images were acquired with the XYZ scanning mode in the $\mathrm{z}$ direction at intervals of $0.1 \mu \mathrm{m}$. A sample was scanned for a period of about 2 to 10 minutes, depending on the thickness of coating film, Z-step, speed, and pixel format of scanning. The following scanning conditions were selected: pinhole size $=67.95 \mu \mathrm{m}$; digital zoom $=$ 1 ; scan area $=386 \times 386 \mu^{2}$; scanning pixel format $=1024 \times 1024$; scanning speed $=400 \mathrm{~Hz}$; laser intensity $=70 \%$; Z-step $=0.1 \mu \mathrm{m}$; and excitation wavelength $=514 \mathrm{~nm}$.

\section{Results and Discussion}

Investigation into the Binder Content of Coating Surface by SEM. Scanning electrical microscopy (SEM) was used to investigate the surface elemental composition of the coating samples. The surface composition in atomic percentage was calculated, and then was presented as energy spectrum. Results are shown in Tables 2. The Al, Si and part of the O signals come from the clay. The main part of the $\mathrm{C}$ signal originates from the SB latex. The amount of carbon originating from each one was calculated with the help of SEM software (Table 2). Table 2 shows the variation of surface composition with different coating solid as found from SEM. In all coating samples investigated, the overall binder content is the same, i.e. binder content of coating surface decreases with coating solid increasing. Low coating solid can result in the adding of carbon content on the coating surface, which means that low coating solid accelerates binder migration towards the coating surface.

Table 2 SEM surface elemental composition in at.\% of coating surface

\begin{tabular}{ccccc}
\hline & $\mathrm{H} 1$ & $\mathrm{H} 2$ & $\mathrm{H} 3$ & $\mathrm{H} 4$ \\
\hline $\mathrm{C}$ & 6.92 & 6.45 & 6.02 & 5.63 \\
$\mathrm{O}$ & 72.51 & 72.15 & 71.68 & 73.10 \\
$\mathrm{Si}$ & 9.22 & 9.18 & 9.82 & 10.14 \\
$\mathrm{Al}$ & 11.21 & 12.06 & 12.33 & 11.00 \\
$\mathrm{~K}$ & 0.14 & 0.14 & 0.15 & 0.13 \\
\hline
\end{tabular}

Characterizing the Z-directional Distribution of Binder by LSCM. Binder was stained with Rhodamine B, so the fluorescent signals detected by LSCM represented the binder exiting. When laser scanned the coating surface, variation of the Fluorescent intensity showed the variation of the amount of the binder in the z-direction. The binder z-directional distribution profiles in Fig. 1 obtained after the samples were measured with the XYZ scanning mode of LSCM in the z-direction 
at intervals of $0.1 \mu \mathrm{m}$. Fig.1 displays the fluorescent intensity distribution in the z-direction (thickness) and illustrates the $z$-directional binder profiles after image processing and analysis using leica software.
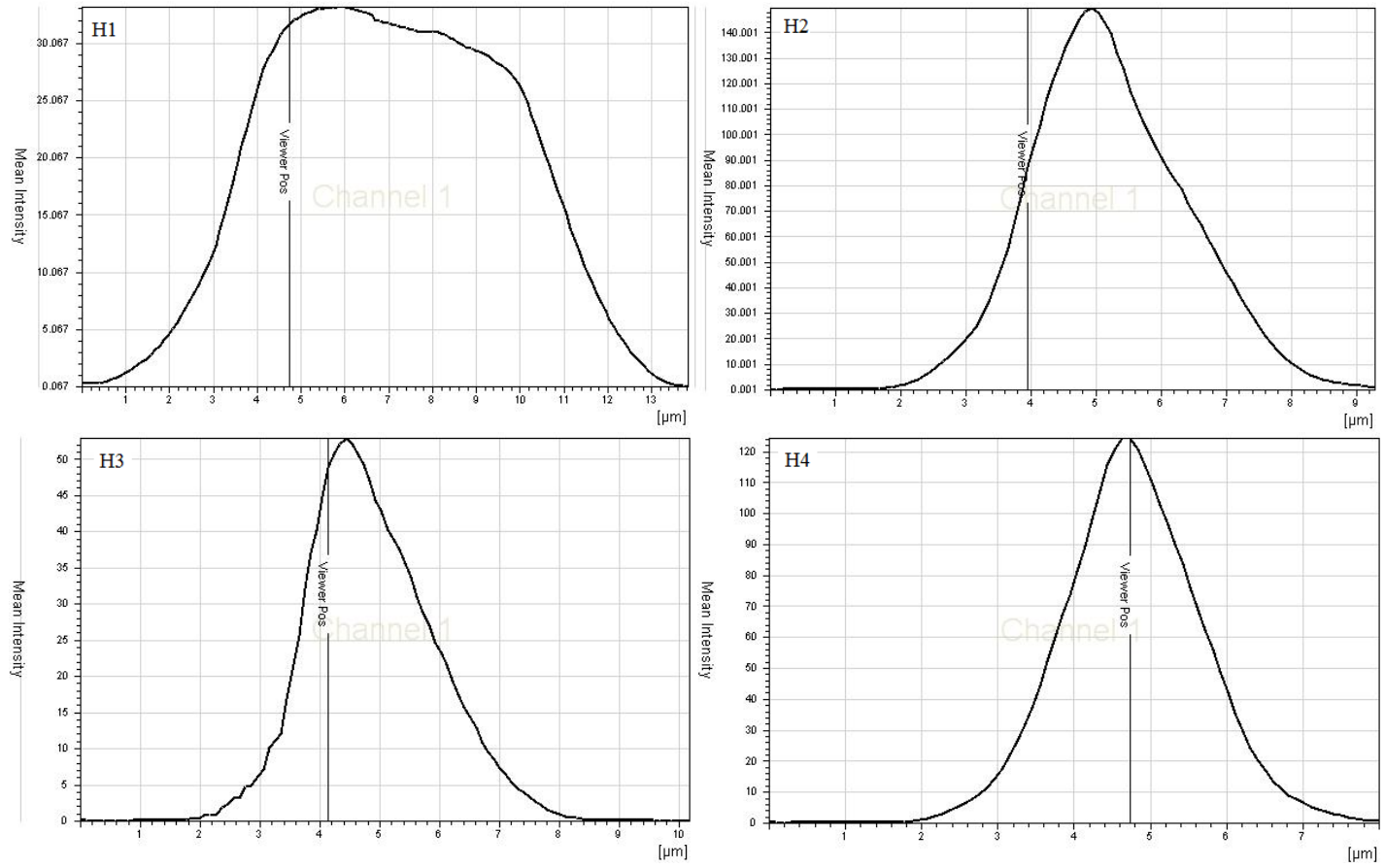

Fig.1. Profiles of the binder z-directional distribution of coating samples which had different solid content.

The fluorescent intensity distribution maps illustrated binder distribution in the z-direction from numerical characterization. Sample $\mathrm{H} 4$ had a quite narrow distribution mainly ranging from $1.8 \mu \mathrm{m}$ to $8 \mu \mathrm{m}$, which implies that sample $\mathrm{H} 4$ had the narrowest binder distribution in these samples. It was clear shown in Fig. 1 that the depth distribution of sample H2 ranging between 1.8 $\mu \mathrm{m}$ and 9.4 $\mu \mathrm{m}$, the depth distribution of sample $\mathrm{H} 3$ ranging between $2.0 \mu \mathrm{m}$ and $8.4 \mu \mathrm{m}$, and sample $\mathrm{H} 1$ had a broadest depth distribution curve mainly ranged from $0.1 \mu \mathrm{m}$ to $13.5 \mu \mathrm{m}$, respectively. It could be concluded that the depth of binder z-directional distribution decrease with the coating solid increasing. The results indicated that the samples differ in fluorescent intensity distribution in z-direction, so the binder z-directional distribution was different due to different coating solid.

The $z$-direction binder profiles suggested that there is a correlation between coating solid and binder distribution. The coating thickness of overall samples was $6 \mu \mathrm{m}$, so the binder must penetrate into the base paper when z-direction length detected by LSCM was larger than $6 \mu \mathrm{m}$. The samples $\mathrm{H} 1$ and $\mathrm{H} 2$ yielded a higher depth value of binder z-directional penetration into base paper compared with the other samples (see Fig. 1). The z-direction length of sample H1 was the largest, which indicated that it was the highest degree of binder migration to the base paper in coating sample H1. There is almost no binder migration towards base paper in sample H4. When sample H1is compared with the others, the findings suggest that low coating solid accelerates water to carry binder migration to coating surface (evaporation) and base paper (absorption) (see Table 2 and Fig. 1). This result was a consequence of that low coating solid had more water in the coating layer, which made water more easily to carry binder to penetrate into the base paper (absorption) and coating surface (absorption). The resulting balance between the two opposite driving forces, absorption and evaporation, governs the movement of the liquid phase within the coating layer as consolidation proceeds, and in turn, it determines the binder spatial distribution in real coated papers $[9,10]$.

\section{Conclusions}

The LSCM can be applied to analyze the degree of binder migration in coated papers. A coated 
paper binder profile with variations in the z- direction variations observed will give an indication of the presence of binder migration. Based on the results presented in this study, the low coating solid was propitious to the preferential movement of the binder to the base paper (absorption) and coating surface (evaporation). Low coating solid seemed to be less desirable because it produced more uneven binder distributions that could potentially result in larger printing defects. More unevenness in the binder distribution can create higher local variations in pore structure, and in turn, spatial variations in absorption that can yield mottling. Appropriate coating solid will resulted in moderate binder distribution and more even changes of binder distribution, which would produce better print quality.

\section{Acknowledgements}

The authors express their gratitude to the Yunnan Province Science and Technology Department (Project KKSY 2012/01059 and KKSY 2012/01051) for financial support.

\section{References}

[1] D.W. Donigian, J. N. Ishley and K. J. Wise: TAPPI J. 80 (5) (1997).

[2] P. Heikkil€a and N. Milosavljevic: Drying Technology 20 (1) (2002) p.211-222.

[3] C. Kugge: J. Pulp Paper Sci. 30 (4) (2004) p.105-111.

[4] R.N.S. Sodhi, L. Sun, M. Sain and R. Farnood: J. Adhesion 84 (3) (2008) p.277-292.

[5] K.G. Hagen: Proceedings of Tappi 1985 Coating Conference, p.131-137.

[6] K.G. Hagen: Tappi 1990 Blade Coating Seminar Notes, Tappi Press, Atlanta, p.125-131.

[7] Y. Ozaki, D.W. Bousfield and S.M. Shaler: Journal of pulp and paper science 31(1) (2005) p.48-52.

[8] Y. Ozaki, D.W. Bousfield and S.M. Shaler: TAPPI J. 5(2) (2006) p.3-8.

[9] G. Engstr“om, M. Rigahl, J. Kline and J. Ahlroos: Tappi J. 5 (1991) p.171-179.

[10] M. Whalen-Shaw, TAPPI Press, Atlanta, 1993. 